\title{
Food Consumption Pattern, Anthropometric Indices and Health Status of Adult Women Aged 30-60 Years in Amucha Autonomous Community, Njaba L.G.A., Imo
} State

\author{
Nkeiruka M. Oly-Alawuba and Okwuchi Charles \\ Department of Nutrition and Dietetics, College of Health Sciences, Imo State University, Owerri 23483, Nigeria
}

\begin{abstract}
The study assessed the anthropometric indices and health status of adult women, aged 30-60 years in relation to their food consumption pattern. The blood sugar level, blood pressure and body mass index of the respondents were determined. A cross sectional survey was used for this study, a clustered and simple random sampling selection technique was used in selecting the samples. Anthropometric indices of weight and height were used to assess the body mass index (BMI), while waist hip ratio (WHR) was used to check for abdominal obesity. Women were considered overweight using world health organization standard (BMI at or above 25), or WHR above 0.85 . Health status was assessed by checking blood pressure and blood sugar levels. Blood pressure was taken twice using Omron sphygmomanometer with appropriate cuff off size and women were classified hypertensive if having a mean blood pressure above 120/80 mmHg. Evolve glucometer was used to check for random blood sugar (RBS) levels, respondents were considered diabetics if they have a random sugar above $140 \mathrm{mg} / \mathrm{dL}$. Food frequency table was used to assess food consumption pattern. Respondents were said to have low consumption if consumed the food 1-3 times per week or if rarely or never consumed. It is moderate if consumed 4-6 times per week and high, if consumed 7 times per week, or daily. Questionnaire covered relevant data such as personal data, demographic data. Result showed that the women mean standard deviations for body mass index (BMI), waist hip ratio (WHR), random blood sugar (RBS), systolic blood pressure (SBP) and diastolic blood pressure (DBP) were 26.29 $\pm 4.1,0.86 \pm 0.34,112.51 \pm$ 23.0, $115.4 \pm 23.0$ and $88.45 \pm 4.3$ respectively. The mean value for BMI was on the overweight region. Bivariate correlation analysis showed that SBP, DBP, RBS and WHR had positive correlation with BMI with confidence level set at 0.05\% $(0.206, p=0.005$; 0.16, $p$ $=0.031 ; 0.081, p=0.0021$ and $0.148, p=0.000$ respectively). WHR also had stronger correlation with RBS $(0.90, p=0.278)$, systolic (0.272, $p=0.002)$, diastolic $(0.21, p=0.045)$. This confirms the assertion that WHR should be used as an indicator of cardiovascular disease not BMI. The survey also reveals that 3.6\% were underweight. $49.0 \%$ normal, $29.9 \%$ overweight and $17.5 \%$ obese. Blood pressure levels were marginal as 30.04\%, 35.4\%, 34.3\% are normal, pre hypertensive and hypertensive respectively. Most of the respondents had normal blood sugar as $91.2 \%$ were normal. Overweight, hypertension and diabetes were prevalent in rural communities as observed in this study. There is a need for routine check of WHR among women in clinical practice and epidemiological surveys, to reduce incidence of cardiovascular diseases. There is a need for promotion of a healthy life style, regular exercise and proper nutrition.
\end{abstract}

Key words: Anthropometric indices, blood pressure, diabetes, overweight, WHR, BMI.

\section{Introduction}

The prevalence of hypertension, diabetes and overweight, as important public health challenges is increasing worldwide [1]. The number of adults with

Corresponding author: Nkeiruka M. Oly-Alawuba, PhD, research fields: community/public health nutrition \& experimental foods. hypertension is predicted to increase by $60 \%$ to a total of 1.56 billion by the year, 2020 [2]. The Framingham study, a famous study for 44 years, estimated that excess body weight accounted for approximately $26 \%$ of cases of hypertension in women and approximately $23 \%$ in men [3]. This could be explained, as overweight individuals have increased fatty tissue, which increases the vascular resistance and in turn 
increases the work the heart has to do in pumping blood throughout the body. Earlier studies attributed the link between overweight and hypertension primarily to haemodynamic factor [4]. Recent evidence indicates that the association is more complex than initially thought [5]. Hypertension and overweight increase the risk of long-term cardio-vascular complications of type 2 diabetes mellitus, including stroke, chronic kidney disease, heart disease, peripheral vascular disease, and death [6]. The relative risk of cardiovascular disease for persons with diabetes is double or more than that of persons without diabetes [7]. Although the absolute risk of cardiovascular disease varies around the world, hypertension and overweight among patients with diabetes mellitus in developing countries are perhaps even more harmful than in high-income countries, where accesses to health care and adequate prevention programs help manage cardiovascular risk factors and can delay complications [8]. Blood pressure reduction has been associated with a decreased risk of type 2 diabetes mellitus related complications, including death, stroke, and the need for retinal photocoagulation [9]. Weight loss helps correct insulin resistance and dyslipidemia found in patients with diabetes mellitus. Deaths from cardiovascular diseases and diabetes are the highest in low- and middle-income countries and the lowest in high-income countries, within all countries, the poorest people are affected the most. Recent recommendations from the American Diabetes Association and the European Association for the Study of Diabetes note that aggressive management of cardiovascular risk factors, which include high blood pressure and obesity, may be even more beneficial in patients with type 2 diabetes mellitus because of their increased risk of cardiovascular morbidity and mortality [10].

Knowing the proportion of the type 2 diabetes mellitus population at additional risk of complications from hypertension and overweight is an important public health measure to determine public and private resource requirements, needed to reduce these risk factors or to care for patients after cardiovascular events. Many epidemiological reports describe separate prevalence rates for hypertension, overweight, and diabetes among the general population [11].

\section{Methodology}

The study was carried out in Amucha Town, Njaba, local government area of Imo State, Nigeria. Amucha is one of the ancient autonomous communities in Njaba local government in Imo State. Villages in the community include Duruobiaku, Duruaku, Duruigwe, Umunnudo, Ummuozi, Aguwa and Duruewuru, close to $85 \%$ of the women are Catholics [12]. The town has one Catholic Parish and three Catholic Stations, located in these villages. Amucha is a rural community with most women income below $\$ 30,000$ per month, and nearly $80 \%$ of its women population are peasant farmers and petty traders and barely $20 \%$ as civil servant [13]. Amucha is heavily reinforced with huge rain forest and valley topography. It enjoys health services from the public health centers in the town and from other neighboring towns [14]. A cross sectional survey design was used for this study. A simple random and clustered sampling selection was used in selecting the samples. This was done by listing out the Catholic Stations in town and knowing the population of women aged 30-60 in each station and they were randomly drawn by using non-replaceable balloting method from the population. "Yes and no" was written on ballot papers, and women that chose yes were used for the survey.

\subsection{Data Collection}

\subsubsection{Questionnaire Administration}

Validated and pre-tested questionnaires were administered to the selected respondents. Information on personal data, demographic characteristics, dietary pattern, health history and food habits was obtained.

\subsubsection{Anthropometric Indices}

Anthropometric indices of body mass index (BMI) and waist-hip ratio (WHR) were used to check for overweight and abdominal overweight respectively. 
Weight and height measurements were used in assessing BMI, while waist and hip circumferences were used to check WHR.

Anthropometric measurements were carried out. The equipment used for the measurement was pre-tested to ensure accuracy. The weighing scale was tested by weighing a substance of known weight. Glucometer was also checked for accuracy by running a control test. BMI and WHR were used to check for overweight and abdominal overweight respectively. Weight and height measurements were used in assessing BMI, while waist and hip circumferences were used to check WHR.

\subsubsection{Weight Measurement}

Weight was taken using Hana mechanical bathroom scale. Foot wear was removed so as to get an accurate weight, respondents were asked to wear light cloth. Wrist watch, bangle, ear rings and chain were all removed. The respondent was asked to mount the scale hands free and placed both sides and head looking forward, and then the weight was read in the nearest kilogram.

\subsubsection{Height Measurement}

The height measurement was taken using calibrated heightometer, the respondent was asked to remove foot wears, stand erect hands placed both sides, and look forward. Head tie, caps and weavon were also removed, and the height was taken in the nearest centimetres.

\subsubsection{BMI}

BMI was used in classifying the person's weight. Women were considered overweight using world health organization standard (BMI at or above 25). The BMI was gotten by dividing the weight in $\mathrm{kg}$, by the height in meter squared.

$$
B M I=\frac{\text { Weight }(\mathrm{kg})}{\text { Height }\left(\mathrm{M}^{2}\right)}
$$

The BMI results were classified using the WHO standard [15] where:

\begin{tabular}{ll}
\hline BMI & Class \\
\hline$<18$ & Underweight \\
$18-24.9$ & Normal \\
$25-29.9$ & Overweight \\
$\geq 30$ & Obesity \\
\hline
\end{tabular}

\subsubsection{WHR}

The waist-hip circumference ratio was taken using WHO STEPS protocol [16], by measuring waist circumference made at the approximate midpoint between the lower margin of the last palpable rib and the top of the iliac crest and the hip circumference measurement was taken around the widest portion of the buttocks. The tape was snug around the body, but not pulled so tight that it is constricting. To ensure accuracy, the subject stands with arms at the sides, feet positioned close together, and weight evenly distributed across the feet and the waist circumference measured at the end of a normal expiration, when the lungs are at their functional residual capacity. Subjects were asked to relax and take a few deep, natural breaths before the actual measurement was made, to minimize the inward pull of the abdominal contents during the waist measurement. The respondent WHR was classified obese if they have waist-hip above 0.85 [17].

The WHR was then in cm calculated by dividing the waist circumference, by the hip circumference.

$$
W H R=\frac{\text { waist circumference }(\mathrm{cm})}{\text { hip cirference }(\mathrm{cm})}
$$

\subsubsection{Food Consumption Pattern}

The food frequency table was used to check for food consumption pattern. The food frequency table consists of a list of food commonly consumed by respondents, and intake of respondents was classified using the following.

(1) Low consumption, 1-3 times per week or if rarely or never consumed;

(2) Moderate consumption, if consumed 4-6 times per week;

(3) High consumption, if 7 times per week or if consumed daily [18].

\subsubsection{Health Status}

Health status was assessed by checking blood pressure and blood sugar levels. Blood pressure was taken twice using Omron sphygmomanometer with appropriate cuff off size and women were classified hypertensive if having a mean blood pressure above 
120/80 mmHg.

Random blood sugar (RBS) was used to assess blood sugar level. Evolve glucometer was used to check for RBS levels, and respondents were considered diabetics if having sugar level at or above $140 \mathrm{mg} / \mathrm{dL}$.

This definition diverges from the standard criterion of a fasting blood glucose concentration of more than $120 \mathrm{mg} / \mathrm{dL}(7.0 \mathrm{mmol} / \mathrm{L})$.

Information gathered from the study was coded into computer software and analyzed using statistical packages for social sciences version 22.0. Results were expressed as frequencies, percentage, means and deviation with significance level set at $p<0.05$. Confidence correlation was done to check for relationships among BMI, WHR, RBS levels, systolic and diastolic blood pressures of respondents.

\section{Results}

Table 1 revealed some personal data of the respondents. In total, $31.49 \%$ of respondents were between the ages of 30-40. A good number of the women surveyed (48.61\%) were between the ages of 51-60 years, which may be as a result of the high rural-urban drift common among younger people. Most of the women surveyed were married (92.27\%) and only $7.73 \%$ were widow. There was no record of divorce or co-habiting. This may be attributed to the fact that, the study was carried out in the Catholic Church, which discourages divorce and co-habiting. A good number of the respondents (53.59\%) were farmers, $21.55 \%$ civil servant and few (2.21\%) were retired. The study also revealed that $41.4 \%$ of the female respondents have 4-6 children, $6.63 \%$ none, $40.33 \%$ have $1-3$ children, which may be attributed to poor formal education.

Table 2 reveals some socio-economic data of the respondents. The result showed that the study area was a low income area. Only $16.02 \%$ earn above thirty thousand naira per month, which could be attributed to poor formal education given to females and other gender inequalities women suffer from. It also showed a decline in income as age increases, which could be attributed to the fact that, low socio-economic status sets in as one gets older [19]. The study also showed that the community has double burden of malnutrition as observed in underweight and overweight occurring at the same population, which may be attributed to inequalities of income and different dietary pattern.

Table 3 showed the distribution of respondents, based on BMI values. Overweight, underweight and obesity occur with percentages of $29.9 \%, 2.6 \%$ and $17.5 \%$ respectively, only $49.0 \%$ were normal. These figures are different from that obtained from the study on market women of Nkwo-Ogutamarket, Oguta LGA of Imo State with percentages of 16\%, 24.7\% and 26\% for underweight, overweight and obesity respectively [20]. The percentage of obese women was lower compared to a study carried in Edo State, by Kragelund et al. [21], although they did not report any age bracket.

Table 2 showed the poor nutrition awareness and poor formal education gave rise to the prevalence of overweight, diabetes and hypertension in the study area; this agrees with other numerous reports like the report of Refs. [22, 23]. This study confirms the assertion that the factors independently and significantly associated with being overweight or obese include, greater age, being literate, and having a high or very low economic status, and a low intake of meat [24].

RBS was also significantly higher in those with elevated WHR than in those with normal WHR. RBS tended to increase significantly with increase in BMI status but overweight individuals tend to have a higher RBS than the obese. Bivariate correlation analysis showed that systolic BP, diastolic BP, RBS and WHR had positive correlation with BMI, $r=0.206$ ( $p=$ $0.005), 0.160$ ( $p=0.031), 0.081(p=0.278)$ and 0.148 ( $p=0.047$ ) respectively. Other studies carried out in other parts of the country have also reported abnormal or elevated WHR among women [25].

Table 4 showed that the women population had a mean random blood sugar of $112.51 \pm 23.93 \mathrm{mg} / \mathrm{dL}$, which is in the normal region. A study carried out in 
Zaria, northern Nigeria by Adamu et al. [26] recorded $85.9+14.7 \mathrm{mg} / \mathrm{dL}$, as its mean sugar level for females with mean age of 36.2.98 [27], reported $22 \pm 17.92$ $\mathrm{mg} / \mathrm{dL}$, although they did not make distinction between values for male and females. In this study, the random blood sugar increased gradually as age increases; this may be as a result of sedentary lifestyle common as age increases. Close to $91.2 \%$ of the population had normal blood sugar levels, $7.2 \%$ were considered diabetics (hyperglycemia) and only 1.5\% of the population had sugar level lower than normal.

Blood pressure is normally expressed in two parameters, the systolic and the diastolic. In Table 4, the result showed the mean systolic blood pressure to be 115 and 88 for the diastolic respectively. Of 35.4\% were pre-hypertensive, $34.3 \%$ hypertensive and $30.4 \%$ normal. World Health Organization [16] reported that higher blood pressure was statistically higher for women who come from rural setting than for women from urban settings $(p=0.016)$. Some authors have reported higher blood pressures values among women in rural communities, Ifeoma [14] reported hypertension to be $28.3 \%$ among adults in Gondar, Northwest Ethiopia. Table 5 shows that there was increase in mean pressure as age increases for both the systolic and diastolic pressure, not only in this study that there has been assertion that blood pressure increases with age but also in numerous study, this phenomena may be explained as a result of sedentary lifestyle or one trying to cope with both family and work stress.

Most studies have postulated that there is a relationship between overweight, diabetes and hypertension. As one gains weight there is a tendency to have high blood pressure and he/she may later become diabetics. Correlation was used to check for association of various variables (BMI, WHR, RBS, systolic and diastolic). In Table 6, the correlation was said to be significant if $p$-value is less than 0.05 . Correlation of BMI with other variables was positively correlated although on the weak side. WHR correlation with other variables was positively correlated and stronger than BMI. RBS was weakly positively correlated with blood pressures. The correlation of BMI with other variables was significant expect RBS, while correlation of WHR and other variables was all significant. Table 7 shows various correlations of BMI, WHR, RBS, and systolic and diastolic blood pressures.

Table 1 Personal characteristics of the respondents.

\begin{tabular}{|c|c|c|c|}
\hline & Variables & Frequency & Percentages (\%) \\
\hline \multirow{3}{*}{ Age } & $30-40$ & 57 & 31.49 \\
\hline & $41-50$ & 36 & 19.89 \\
\hline & $51-60$ & 88 & 48.61 \\
\hline \multirow{4}{*}{ Marital status } & Married & 167 & 92.27 \\
\hline & Widowed & 14 & 7.73 \\
\hline & Divorced & 0 & 0 \\
\hline & Widow & 0 & 0 \\
\hline \multirow{5}{*}{ Occupation } & Civil servant & 39 & 21.55 \\
\hline & Farmer & 97 & 53.59 \\
\hline & Trader & 24 & 13.26 \\
\hline & Artisans & 17 & 9.39 \\
\hline & Retired & 4 & 2.21 \\
\hline \multirow{5}{*}{ Number of children } & None & 12 & 6.63 \\
\hline & $1-3$ & 73 & 40.33 \\
\hline & $4-6$ & 75 & 41.4 \\
\hline & $>6$ & 21 & 11.6 \\
\hline & Total & 181 & 100 \\
\hline
\end{tabular}


Table 2 Socio-economic data of the respondent.

\begin{tabular}{|c|c|c|c|}
\hline & Options & Frequency & Percentage (\%) \\
\hline \multirow{5}{*}{ Level of income } & 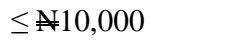 & 58 & 32.04 \\
\hline & $\$ 10,500-15,000$ & 41 & 32.65 \\
\hline & $\$ 15,500-20,000$ & 25 & 13.81 \\
\hline & $\$ 20,500-30,000$ & 28 & 15.47 \\
\hline & $\$ 30,000$ & 29 & 16.02 \\
\hline \multirow{4}{*}{ Level of education } & Primary & 67 & 37.02 \\
\hline & Secondary & 68 & 54.14 \\
\hline & Tertiary & 7 & 3.87 \\
\hline & None & 9 & 4.97 \\
\hline \multirow{7}{*}{ Sources of food consumed } & Farm & 17 & 9.39 \\
\hline & Local market & 47 & 25.97 \\
\hline & Imported & 0 & 0 \\
\hline & Market/imported & 3 & 1.66 \\
\hline & Farm/imported & 0 & 0 \\
\hline & Farm/market & 110 & 60.77 \\
\hline & Gift & 4 & 2.21 \\
\hline
\end{tabular}

Table 3 Distribution of respondents based on BMI values.

\begin{tabular}{lll}
\hline BMI values & Frequency & Percentage (\%) \\
\hline Underweight & 7 & 3.6 \\
Normal & 87 & 49.0 \\
Overweight & 55 & 29.9 \\
Obese & 32 & 17.5 \\
Total & 181 & 100 \\
\hline
\end{tabular}

Table 4 Mean standard deviation of the levels of blood pressure, body mass index, waist-hip ratio and random blood sugar.

\begin{tabular}{lllll}
\hline Variables & $30-40$ & $41-50$ & $51-60$ & Total \\
\hline BMI & $23.05 \pm 4.0$ & $24.62 \pm 5.57$ & $26.31 \pm 5.3$ & $26.29 \pm 4.1$ \\
WHR & $085 \pm 0.05$ & $0.867 \pm 0.06$ & $0.86 \pm 0.05$ & $0.86 \pm 0.34$ \\
RBS & $103.4 \pm 7.6$ & $108.01 \pm 12.7$ & $111.23 \pm 14.7$ & $112.51 \pm 23.93$ \\
Systolic & $110.9 \pm 23$ & $113.4 \pm 17.7$ & $117.0 \pm 28$ & $115.48 \pm 23.65$ \\
Diastolic & $79 \pm 9.6$ & $81.23 \pm 17.8$ & $82.42 \pm 14.0$ & $88.45 \pm 4.39$ \\
\hline
\end{tabular}

Table 5 Classification of respondents based on blood pressure values.

\begin{tabular}{lll}
\hline Blood pressure & Number & Percentage (\%) \\
\hline Normal & 55 & 30.4 \\
Pre-hypertension & 64 & 35.4 \\
Hypertension & 62 & 34.3 \\
Total & 181 & 100 \\
\hline
\end{tabular}

Table 6 Correlation of BMI, WHR, RBS and blood pressure values.

\begin{tabular}{llllll}
\hline Variables & BMI & WHR & RBS & Systolic & Diastolic \\
\hline BMI & 1 & & & & \\
WHR & $0.148(0.047)$ & 1 & & & \\
RBS & $0.081(0.278)$ & $0.90^{*}(0.001)$ & 1 & & \\
Systolic & $0.206(0.005)$ & $0.272^{*}(0.002)$ & $-0.012^{*}(0.875)$ & 1 & 1 \\
Diastolic & $0.16 *(0.031)$ & $0.21 *(0.045)$ & $-0.024^{*}(0.75)$ & $0.75(0.001)$ & \\
\hline
\end{tabular}

Figures in bracket are $p$-values. The correlation is significant if $p$-value $<0.05$;

* Figures are not significant. 
Table 7 Distributionof respondents based on the blood sugar.

\begin{tabular}{lll}
\hline Random blood sugar & Number & Percentage (\%) \\
\hline Normal & 165 & 91.2 \\
Hyper-glycaemia & 13 & 7.2 \\
Hypoglycaemia & 3 & 1.5 \\
Total & 181 & 100 \\
\hline
\end{tabular}

Table 6 also showed the relationship between blood pressure and the BMI of the respondents. Diastolic and systolic blood pressures increased significantly with increasing BMI status. RBS was also significantly higher in those with elevated WHR than in those with normal WHR. RBS tended to increase significantly with increase in BMI status but overweight individuals tend to have a higher RBS than the obese. Bivariate correlation analysis showed that systolic BP, diastolic BP, RBS and WHR had positive correlation with BMI, $r=0.206$ ( $p=0.005), 0.160(p=0.031), 0.081(p=$ $0.278)$ and $0.148(p=0.047)$ respectively.

\section{Conclusion}

Body mass index (BMI) and waist-hip ratio (WHR) were positively correlated with random blood sugar (RBS) and blood pressure in the study area. The women were therefore at risk of overweight and its related health conditions (hyperglycaemia and hypertension). The increase in body weight, sugar level may be as a result of dietary, lifestyle and genetic factors. Overweight, diabetes and hypertension in women lead to escalating health cost among women. These conditions were formally seen as problems of affluence and as such, have prevalence in high income areas but this study showed that they can occur in rural communities. Population based preventive measures for the control of the diabetes mellitus, hypertension and overweight epidemic must therefore include avoidance of adiposity through physical activity and regulated caloric intake. This study revealed a more significant positive correlation of WHR with other variables, this therefore, confirms the assertion that WHR and not BMI, should be used as a more reliable indicator of cardiovascular diseases during clinical and epidemiological survey.

\section{Reference}

[1] World Health Organization. 2000. Obesity Preventing and Managing the Global Epidemic. WHO Technical Report Series Geneva 1999.

[2] World Health Organization. 2003. Fact Sheet 317 Geneva.

[3] Kannel, W. B., and McGee, D. L. 1979. "Diabetes and Cardiovascular Risk Factors: Framingham Study.” Circulation 59: 8-13.

[4] World Health Organization. 2000. (Obesity; Preventing and Managing the Global Epidemic.) WHO technical report series No: 894. Geneva.

[5] Onupuu, S., and Anads, S. 2001. "Global Burden of Cardiovascular Disease. Part 1.” Circulation 104: 2746-53.

[6] Inzucchi, S. E., Bergenstal, R. M., and Buse, J. B. 2012. "A Position Statement American Diabetes Association (ADA), European Association for the Study of Diabetes (EASD).” Diabetic Care 32: 420.

[7] Centre for Disease Control. 2004. "Prevalence of Overweight and Obesity among Adults with Diagnosed Diabetes-United States, 1988-1994 and 1999-2002.” Morbidity and Mortality Weekly Report 45: 1066-8.

[8] Nzeagwu, O. C., and Okorocha, C. G. 2012. “Assessment of Nutritional Vulnerability of Hospitalized Elderly in University of Nigeria Teaching Hospital Enugu and Federal Medical Center (FMC) Umuahia, Abia State.” Journal of Dietitians Association of Nigeria 3: 21-35.

[9] U.S. Department of Agriculture and U.S. Department of Health and Human Service. 2005. Dietary Guidelines for America 2005. Washingthon D.C.

[10] Mayo Foundation. 2014. "Mayo Clinic.” Accessed Nov. 13, 2016. http//www.Mayoclinic.org.

[11] Innocent, I., Chuku A., Collins, J., et al. 2013. "Prevalence of Overweight and Obesity in Adult Nigerians-A Systematic Review.” Diabetes, Metabolic Syndrome and Obesity 6: 43-7.

[12] Dimkpa. 2007. “Assessment of Nutritional Intake, Body Mass Index, Activity Levels and Glycaemic Control in Patient with Type 2 Diabetes.” Journal of Nutrition Science.

[13] Jafar, T. H., Chaturvedi, N., and Pappas, G. 2006. "Prevalence of Overweight and Obesity and Their Association with Hypertension and Diabetes Mellitus in an Indo-Asian Population.” Canadian Medical 
Association Journal 175 (9): 1072-3.

[14] Ifeoma, E. 2015. Prevalence of Overweight and Obesity among Nkwo-Oguta Market in Oguta LGA. Project Research, Chapter 5, p. 54.

[15] Abiodun, O., Jagun, O., Olu-Abiodun, O., and Sotunsa, J. “Correlation between Body Mass Index, Waist Hip Ratio, Blood Sugar Levels and Blood Pressure in Apparently Healthy Adult Nigerians." IOSR Journal of Dental and Medical Sciences (IOSR-JDMS) 13 (11): 56-61.

[16] World Health Organization. 2012. Waist Circumference and Waist-Hip Ratio. Report of a WHO Expert Consultation, Geneva, December 8-11, 2008.

[17] World Health Organization. 2003. Obesity: Preventing and Managing the Global Epidemic. Report of a WHO Convention. World Health Organization Technical Report Series.

[18] Akilew, A., Tadesse, A., Shitaye, A., and Berihum, M. 2012. "Prevalence and Associated Factors of Hypertension among Adults in Gondar, Northwest Ethiopia: A Community Based Cross Sectional Study.” BMC Cardiovascular Disorders 12: 113

[19] Dowling, F., and Pi-Sunyer, X. F. 1993. "Race-Dependent Health Risks of Upper Body Obesity.” Diabetes 42: 537-43.

[20] National Institute of Health (NIH), National Heart, Lungs and Blood Institute. 1992. Clinical Guidelines on the Identification, Evaluation and Treatment of Overweight and Obesity in Adults. Department of Health and Human Services, Public Health Services (PHS).
[21] Kragelund, C., Hassager, C., Hildebrandt, P., Torp-Pedersen, C., and Kober, L. 2005. "Impact of Obesity on Long-Term Prognosis Following Acute Myocardial Infarction.” International Journal of Cardiology 98: 123-31.

[22] Igbokwe, C. C., and Ukwuma, M. C. 2013. "Incidence of Hypertension among Pregnant Women in Enugu East Local Government Area of Enugu State (2009-2012).” International Journal of Research in Arts and Social Sciences 6: 302-43.

[23] Dietz, W. H. Jr. 1986. "Prevention of Childhood Obesity.” Pediatric Clinic of North America 33 (4): 823-33.

[24] Dietz, W. H. 1998. "Childhood Weight Affect Adult Morbidity and Mortality." Journals of Nutrition 128: 411-4.

[25] International Diabetes Federation. 2013. “A Prevalence Estimate of Diabetes Mellitus, 2010 by Country Percent of Adult Population with Diabetes, Ranked Highest to Lowest \%.” Accessed Nov. 13, 2016. http://www.allcountries.org/ranks/diabetes_prevalence_c ountry_ranks.html.

[26] Adamu, B., Geoffrey, C. O., Bala, G. S., Ibrahim, S. A., Sani, H., and Tambaya, M. A. 2006. "Relationship between Random Blood Sugar and Body Mass Index in an African Population.” International Journal of Diabetes and Metabolism 14: 144-5.

[27] Janghorbani, M., Hedley, A. J., and Jones, R. B. 1991. “Is the Association between Glucose Level and 'All Causes' and Cardiovascular Mortality Risk Dependent on Body Mass Index?” Irish Medical Journal 6: 205-12. 